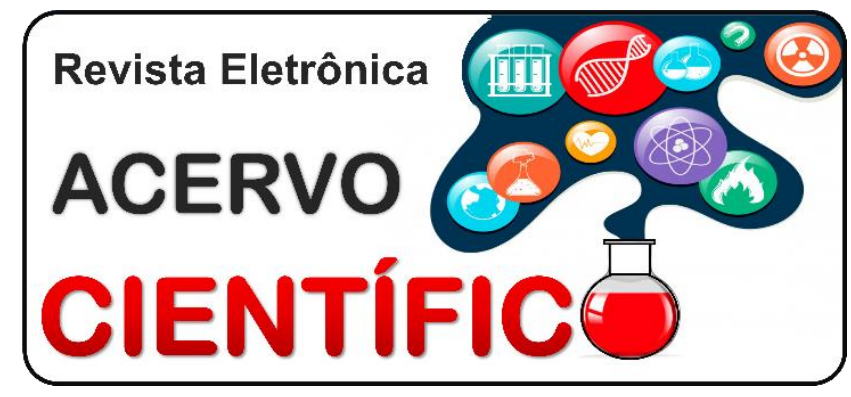

ESTUDO DE CASO

Recebido em: 10/2020

Aceito em: 11/2020

Publicado em: 11/2020

\title{
Estenose de cólon transverso com realização de transversectomia: relato de caso
}

\author{
Transverse colon stenosis with transversectomy performance: case report
}

Estenosis de colon transverso con rendimiento de transversectomía: reporte de caso

Henrique Amorim Santos ${ }^{1 *}$, Amanda Karolyne Batista Ferreira ${ }^{1}$, Isabella Amorim Santos ${ }^{2}$, Mateus Fernandes Alves dos Reis ${ }^{1}$, Michelle Maia Garcez ${ }^{1}$, Roberto Oliveira Argondizzi ${ }^{1}$, Mirelli Leonardi laquinto ${ }^{3}$, Karen Pereira Rocha ${ }^{1}$.

Resumo: A obstrução intestinal ocorre devido a uma diversidade de anomalias congênitas ou adquiridas, sendo a estenose intestinal uma, embora rara, importante causa de obstrução intestinal em recém-nascidos. Contudo, o diagnóstico pode ser retardado visto que nenhum dos sintomas de maior prevalência são patognomônicos da condição e a etiologia da estenose ainda não é bem entendida. Neste trabalho, relatamos o caso de um recém-nascido do sexo feminino que apresentou, desde a primeira semana de vida, quadros recorrentes de vômitos e distensão abdominal, associados a ausência de evacuação. Inicialmente, após enema opaco, foi levantado como hipótese diagnóstica a Doença de Hirschsprung, mas após a manutenção do quadro mesmo com tratamento conservador, foi realizada laparotomia e o caso revelou-se uma estenose de transverso, sendo adotado a correção cirúrgica através da transversectomia com ileostomia e fístula cutânea protetora. Desse modo, o caso foi apresentado com a finalidade de alertar para a necessidade de se considerar os diversos diagnósticos diferenciais na elaboração do pensamento clínico e, nesse caso, a necessidade de se considerar a estenose de intestino na elaboração de diagnósticos diferenciais em lactentes com quadro de constipação crônica.

Palavras-chave: Obstrução intestinal, Cólon transverso, Colectomia.

Abstract: Intestinal obstruction occurs due to a variety of congenital or acquired abnormalities, with intestinal stenosis being one, although rare, important cause of intestinal obstruction in newborns. However, the diagnosis can be delayed since none of the most prevalent symptoms are pathognomonic of the condition and the etiology of the stenosis is still not well understood. In this work, we report the case of a newborn female who presented, since the first week of life, recurrent vomiting and abdominal distension, associated with the absence of evacuation. Initially, after barium enema, Hirschsprung's Disease was raised as a diagnostic hypothesis, but after maintaining the condition even with conservative treatment, a laparotomy was performed and the case revealed as a transverse stenosis, with surgical correction being adopted through transversectomy with protective ileostomy and skin fistula. Therefore, the case was presented with the purpose of alerting the need to consider the several differential diagnoses in the elaboration of clinical thinking and, in this case, the need to consider the intestinal stenosis in the elaboration of differential diagnoses in infants with chronic constipation.

Keywords: Intestinal obstruction, Colon transverse, Colectomy.

Resumen: La obstrucción intestinal ocurre debido a una variedad de anomalías congénitas o adquiridas, siendo la estenosis intestinal, aunque rara, una causa importante de obstrucción intestinal en los recién

1 Universidade Federal do Triângulo Mineiro (UFTM), Uberaba - MG. *E-mail: henriqueamorims@gmail.com

2 Universidade de Franca (UNIFRAN), Franca - SP.

3 Universidade Federal de Mato Grosso do Sul (UFMS), Três Lagoas - MS. 
nacidos. Sin embargo, el diagnóstico puede ser demorado ya que ninguno de los síntomas más prevalentes es patognomónico de la enfermedad y aún no se conoce bien la etiología de la estenosis. En este trabajo presentamos el caso de una recién nacida, que desde la primera semana de vida presentó vómitos recurrentes y distensión abdominal, asociados a la ausencia de evacuación. Inicialmente, después de realizar un enema de bario, se planteó como hipótesis diagnóstica la Enfermedad de Hirschsprung, pero tras mantener el cuadro,incluso con tratamiento conservador, se realizó una laparotomía y el caso reveló una estenosis de colon transverso, adoptando la corrección quirúrgica mediante transversostomía con ileostomía y fístula cutânea protectora. Así, el caso se presentó con el propósito de alertar sobre la necesidad de considerar los diversos diagnósticos diferenciales en la elaboración del pensamiento clínico y en este caso, la necesidad de considerar la estenosis intestinal en la elaboración de diagnósticos diferenciales en recién nacidos con estreñimiento crónico.

Palabras clave: Obstrucción intestinal, Colon transverso, Colectomía.

\section{INTRODUÇÃO}

A obstrução intestinal ocorre devido a uma diversidade de anomalias congênitas ou adquiridas, como atresia e estenose intestinal, pâncreas anular, íleo meconial, má rotação intestinal, doença de Hirschsprung, neoplasias, entre outras causas. Dessa forma, a estenose colônica é uma, embora rara, importante causa de obstrução intestinal em recém-nascidos (EKENZE SO, et al., 2019; HAJIVASSILIOU CA, 2003; XIE X, et al., 2018).

A estenose colônica congênita é uma variante da atresia intestinal colônica, com gravidade variável, dependendo do estreitamento do lúmen associado. Trata-se de uma anomalia congênita rara do trato gastrointestinal, com incidência relatada de 1 a cada 40.000 nascimentos, onde há uma malformação intestinal, na qual o segmento proximal dilatado está em continuidade com o segmento distal, através de um curto segmento, rígido e estreito, com lúmen mínimo e mesentério íntegro (EKENZE SO, et al., 2019; GALVÁN-MONTAÑO A, et al., 2010; UPADHYAYA VD, et al., 2020).

A estenose colônica se apresenta clinicamente com êmese biliosa, distensão abdominal, falha na passagem de mecônio e fezes pelo ânus e dificuldade para ganhar peso (EKENZE SO, et al., 2019; ELISA Z, et al., 2016; GALVÁN-MONTAÑO A, et al., 2010; HAMID R, et al., 2015). Contudo, os sinais e sintomas de estenose colônica congênita são inespecíficos, podendo fazer parte de diversas outras afecções do trato gastrintestinal, fazendo com que o diagnóstico definitivo da estenose colônica seja, na maioria das vezes, durante a cirurgia (EKENZE SO, et al., 2019).

Nesse contexto, os exames complementares devem ser utilizados para auxiliar no diagnóstico da estenose colônica, podendo ser utilizados raio-x contrastado via retal (enema opaco) e colonoscopia, sendo o tratamento definitivo cirúrgico, através de laparotomia ou laparoscopia (ELISA Z, et al., 2016; EKENZE SO, et al., 2019; HAMID R, et al., 2015; MIRZA B, et al., 2012). Nesse sentido, o manejo de neonatos com essa condição melhorou em décadas recentes devido a aperfeiçoamentos em terapia intensiva neonatal, técnica operatória, uso de nutrição parenteral total e anestesia neonatal (EKENZE SO, et al., 2019).

Dessa forma, o objetivo deste trabalho é relatar um caso de estenose colônica, com o acometimento do cólon transverso em um neonato que se apresentava com quadro de distensão abdominal, constipação intestinal e vômitos em grande quantidade em um hospital, elencando as dificuldades no manejo e no diagnóstico desses pacientes.

\section{DETALHAMENTO DO CASO}

Recém-nascido do sexo feminino, parto cesáreo, pré-termo, com presença de corioamnionite e bolsa rota com mais de 24 horas, com idade gestacional de 30 semanas e 3 dias, gestação com pré-natal incompleto (apenas 4 consultas), peso ao nascimento de $1580 \mathrm{~g}$ e APGAR 8/9, acianótico, com choro vigoroso e tônus em flexão. Ainda na sala de parto, o recém-nascido recebeu as medidas de assistência, sendo posicionado, envolto em saco plástico e colocado touca. Em seguida, foi realizado aspiração de vias 
aéreas superiores, mantendo frequência cardíaca acima de 100 bpm e respiração regular. Contudo, posteriormente, evoluiu com quadro de desconforto respiratório, sendo iniciado o uso de CPAP, com ventilador manual com PEEP de 6, evoluindo com melhora gradual da saturação. Desse modo, foi encaminhada à UTI neonatal em incubadora de transporte com fração inspirada de oxigênio (FiO2) de $100 \%$, onde foi colocado em BIPAP e iniciada antibioticoterapia profilática com ampicilina e gentamicina. A paciente evoluiu com melhora progressiva do padrão respiratório e após 1 dia sob BIPAP se estabilizou em ar ambiente.

Com 5 dias de vida, o recém-nascido apresentou distensão abdominal com melhora rápida e espontânea em 24 horas e, posteriormente, com 18 dias de vida, foi solicitada avaliação da cirurgia pediátrica devido à ausência de evacuação há quinze dias e quadro de vômitos em grandes quantidades associados à nova distensão abdominal há oito dias, que cessou após a introdução de dieta zero por 24 horas. Após o retorno da dieta oral, a paciente evoluiu com novo episódio de distensão abdominal e manutenção do quadro de vômitos. Dessa forma, foi realizado exame de enema opaco (Figura 1), no qual apresentou realce somente até o cólon descendente e evidenciou afilamento concêntrico de bordas regulares da porção distal do reto por cerca de 1 a $2 \mathrm{~cm}$, sem significativa dilatação a montante, além de ausência de áreas de estenose / obstrução, sendo aventada então, hipótese diagnóstica de Doença de Hirschsprung. Assim, com o propósito de confirmar a suspeita de Doença de Hirschsprung, a paciente foi submetida à biópsia retal, com amostras de espessura total localizadas a 3,10 e $15 \mathrm{~cm}$ da borda anal e foi instaurado tratamento conservador, com dieta oral (leite materno por ordenha) conforme aceitação e realizado de dilatação retal com vela de Hegar $\mathrm{n}^{\circ} 8$ diariamente. $\mathrm{O}$ laudo anatomopatológico evidenciou parede retal dentro dos limites de normalidade histológica e presença de células ganglionares em plexos mioentéricos e submucosos de aspecto imaturo, sendo descartada, dessa forma, a suspeita de Doença de Hirschsprung.'

Figura 1 - Exame de enema opaco para a avaliação colônica.

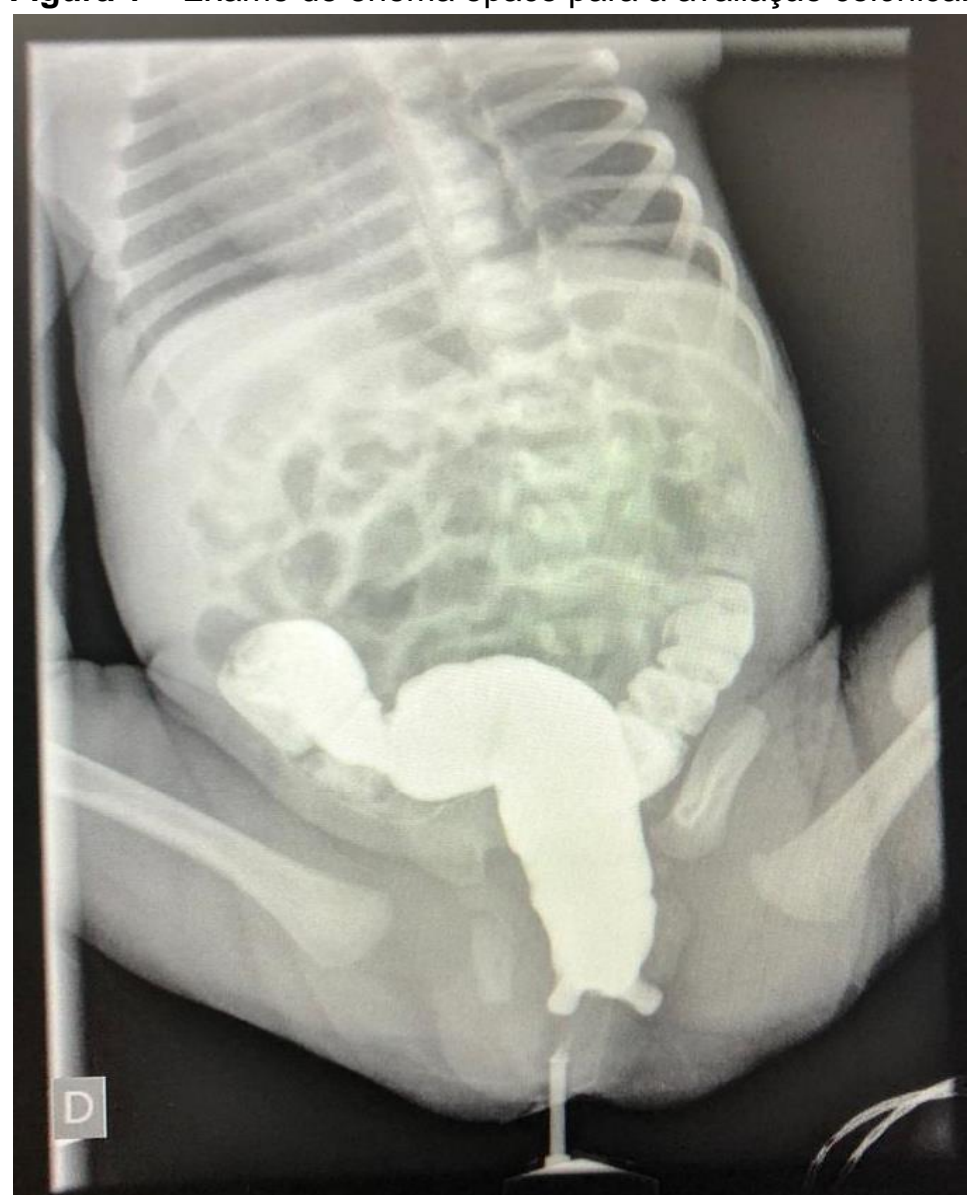

Fonte: Santos HA, et al., 2020. 
A paciente evoluiu com manutenção da distensão abdominal e intolerância a progressão da dieta, apresentando náuseas e vômitos, além de suboclusão intestinal. Diante da persistência do quadro clínico, e da ausência de exames complementares que pudessem ser utilizados para elucidar o quadro, foi indicada laparotomia exploratória transversa supraumbilical direita, sendo encontrado no inventário da cavidade, estenose de cólon transverso de aproximadamente $3 \mathrm{~cm}$, sem sinais inflamatórios e/ou infecciosos. Além disso, foi corrido alças, no qual não se evidenciou outros pontos estenosantes, tampouco má rotação intestinal.

Desse modo, foi realizado transversectomia segmentar de aproximadamente $5 \mathrm{~cm}$ com confecção de colostomia em dupla boca, devido às condições clínicas da paciente, como baixo peso $(1,5 \mathrm{~kg})$ e da grande desproporção entre as alças intestinais. A cirurgia ocorreu sem intercorrências e sem grande perda de sangue. A análise histopatológica do segmento retirado evidenciou uma mucosa lisa e acastanhada, com pregueamento em parte usual, em parte ausente.

Além disso, à macroscopia, havia áreas esbranquiçadas em uma das extremidades e mais endurecida do que as demais. Ademais, à microscopia óptica, o segmento apresentou estenose, com hiperplasia da camada muscular própria, ulceração da mucosa associada a tecido de granulação, fibrose e reação inflamatória crônica produtiva, confirmando o diagnóstico de estenose de cólon transverso (Figura 2).

Figura 2 - Microscopia óptica do segmento de cólon transverso.

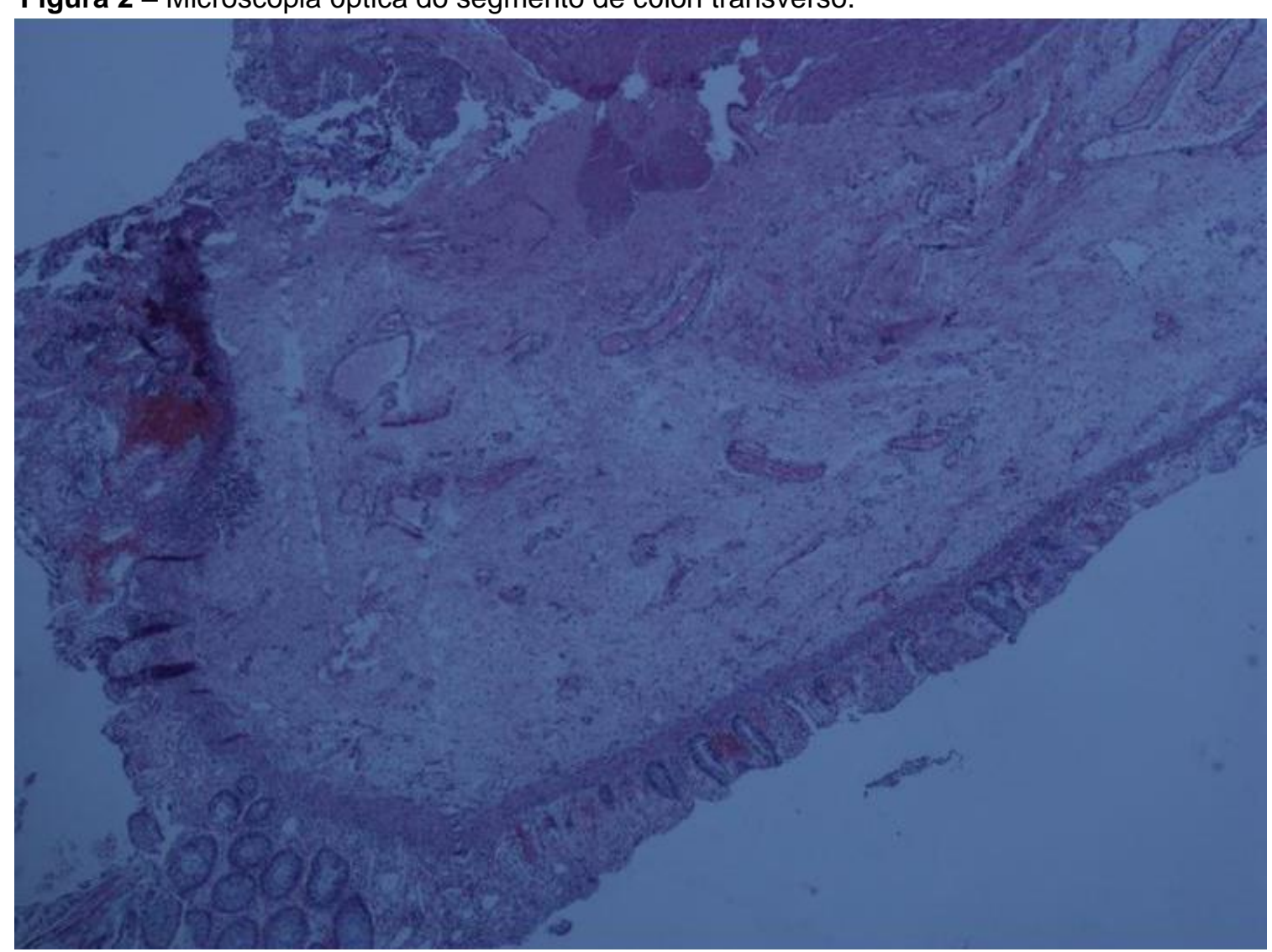

Fonte: Santos HA, et al., 2020.

Assim, a paciente foi encaminhada à UTI neonatal para cuidados pós operatórios. Após a realização da cirurgia, a paciente apresentou boa evolução, com liberação da dieta por sonda oro-gástrica, com boa aceitação, tolerando progressão até dieta-alvo. Ademais, a colostomia se manteve em bom estado e funcionante durante todo o período. Dessa forma, devido à boa evolução, a paciente foi transferida para a enfermaria para continuação dos cuidados. No $12^{\circ}$ dia de pós-operatório, a paciente recebeu alta hospitalar, com orientação de retorno ambulatorial e programação de reconstrução do trânsito intestinal. 


\section{DISCUSSÃO}

A estenose colônica é uma rara causa de obstrução intestinal, podendo ser congênita ou adquirida, e sua apresentação pode se dar tanto no período neonatal quanto na primeira infância e, menos comumente, em crianças mais velhas (EKENZE SO, et al., 2019; GALVÁN-MONTAÑO A, et al., 2010; HAMID R, et al., 2015). A etiologia da estenose colônica é desconhecida, mas o desenvolvimento da doença tem sido associado a acidentes vasculares mesentéricos fetais causados por torções intestinais, hérnia interna, intussuscepção, volvo e uso materno de cocaína. Quanto aos casos adquiridos, estão associados à ocorrência após quadro de Enterocolite Necrotizante $(E C N)$, infecções congênitas pelo Citomegalovírus e à colite decorrente do uso de antibióticos (EKEMA G, et al., 2006; EKENZE SO, et al., 2019; UPADHYAYA VD, et al., 2020; XIE X, et al., 2018).

Com relação ao acometimento do cólon, a apresentação clínica pode incluir êmese biliosa, distensão abdominal, falha na passagem de mecônio e fezes pelo ânus e dificuldade para ganhar peso (EKENZE SO, et al., 2019; ELISA Z, et al., 2016; GALVÁN-MONTAÑO A, et al., 2010; HAMID R, et al., 2015). Assim, o quadro da paciente em questão condiz com a descrição na literatura, apesar da dificuldade de visualização da estenose no enema baritado. No entanto, o diagnóstico pode ser atrasado visto que nenhum dos sintomas de maior prevalências são patognomônicos da condição, podendo estar associados à outras anormalidades, como a Doença de Hirschsprung, ECN, atresia distal do intestino delgado e má rotação intestinal, que podem estar presentes na mesma faixa etária, podendo ser incluídas na elaboração de diagnósticos diferenciais (EKENZE SO, et al., 2019).

A apresentação da estenose colônica pode se dar no período neonatal, na primeira infância ou até mesmo em crianças mais velhas, e os fatores que determinam o momento da apresentação não são completamente conhecidos. Entretanto, o momento da apresentação pode estar relacionado ao fato de a estenose ser congênita ou adquirida e à gravidade da estenose (BHARTI LK, et al., 2017; EKENZE SO, et al., 2019; GALVÁN-MONTAÑO A, et al., 2010; HAMID R, et al., 2015).

O enema de bário auxilia na definição da localização da estenose colônica, delineando um cólon distal de calibre reduzido e cólon proximal distendido, além de permitir a diferenciação da atresia, na qual há um ponto de corte entre um cólon distal preenchido com contraste e intestino proximal dilatado cheio de ar luminal. Ademais, a realização de colonoscopia também pode auxiliar no diagnóstico da estenose de colón, podendo revelar uma mudança abrupta de calibre na área afetada do cólon (EKENZE SO, et al., 2019; ELISA Z, et al., 2016; GALVÁN-MONTAÑO A, et al., 2010; MIRZA B, et al., 2012). Na paciente, foi feito raio$x$ contrastado via retal, que evidenciou afilamento concêntrico de bordas regulares da porção distal do reto por cerca de 1 a $2 \mathrm{~cm}$, sem significativa dilatação a montante, ainda com a hipótese inicial de Doença de Hirschsprung. Apesar de o enema opaco ser um dos melhores exames para auxiliar no diagnóstico da estenose colônica, neste caso, como não foi contrastado todo cólon, não foi contemplado o local da estenose, não permitindo, assim, o completo estudo do cólon e sendo um dos motivos para a indicação da laparotomia exploratória.

O número de casos de obstrução intestinal que são identificados antes do nascimento aumentou como resultado do uso rotineiro de monitoramento ultrassonográfico fetal. A ultrassonografia pré-natal auxilia melhor no diagnóstico da atresia intestinal, já que pode identificar a presença de polidrâmnio materno e distensão do estômago e duodeno com líquido amniótico deglutido. O exame precoce (com no mínimo 18 semanas de gestação) associado ao diagnóstico pré-natal com o reconhecimento precoce e intervenção cirúrgica imediata está associado à melhor prognóstico e redução das complicações metabólicas. Entretanto, a ultrassonografia pré-natal muitas vezes pode não detectar a presença de estenoses colônicas. No caso da paciente em questão, a mãe não realizou corretamente o acompanhamento pré-natal e não atingiu o total de 6 consultas preconizadas pelo Ministério da Saúde, o que dificultou a possibilidade de um diagnóstico precoce e, assim, o diagnóstico da condição só foi realizado durante laparotomia exploratória.

O tratamento definitivo da condição, seja congênita ou adquirida, é cirúrgico. A estenose pode ser única ou múltipla, podendo afetar cólon ascendente, transverso, descendente ou sigmoide (EKENZE SO, et al., 2019; HAJIVASSILIOU CA, 2003). A abordagem cirúrgica pode ser aberta ou por via laparoscópica e 
envolve a ressecção do segmento estenosante e anastomose primária, com relação à afecção do cólon direito. Na estenose do cólon esquerdo, pode se associar a realização de ileostomia protetora proximal, após a ressecção e anastomose (EKENZE SO, et al., 2019; ELISA Z, et al., 2016; HAMID R, et al., 2015; MIRZA B, et al., 2012).

No caso, a estenose afetou o cólon transverso, sendo realizada transversectomia associada à ileostomia com fístula cutânea protetora, durante a laparotomia exploratória, com boa evolução por parte da paciente, sem intercorrências. As complicações associadas ao período pós-operatório incluem infecção da ferida cirúrgica, prolapso de colostomia, íleo prolongado e obstrução intestinal adesiva tardia. Nesse sentido, os avanços no suporte intraoperatório e cuidados pós-operatórios, como a UTI neonatal, levaram a altas taxas de sobrevivência e redução significativa de possíveis complicações (EKENZE SO, et al., 2019).

A estenose colônica, embora rara, é uma importante causa de obstrução intestinal na primeira infância, resultando em diferentes sintomas como a distensão abdominal e constipação, apresentados no caso do recém-nascido aqui relatado. Deste modo, a busca pelo diagnóstico diferencial sempre deve ser feita, uma vez que os sintomas podem fazer parte de inúmeras afecções e o correto diagnóstico é imprescindível para a instituição do tratamento precoce. Assim, durante o desenvolvimento do pensamento clínico no processo diagnóstico, se torna imprescindível suspeitar de estenose colônica como diagnóstico diferencial em recém nascidos com distensão abdominal crônica ou recorrente e, a partir dos exames complementares, confirmar o diagnóstico para instituir o tratamento mais adequado.

\section{REFERÊNCIAS}

1. BHARTI LK, et al. Unusual Presentation of Congenital Stenosis of Sigmoid Colon: Case Report. Journal of Surgical Techniques and Procedures, 2017; 1(1):1002.

2. EKEMA G, et al. Colonic stricture mimicking Hirschsprung's disease: a localized cytomegalovirus infection. J Pediatr Surg, 2006;41(4):850-2.

3. EKENZE SO, et al. Chronic bowel obstruction from colonic stenosis in early infancy-A report of two cases. Malawi Medical Journal, 2019; 31(1):82-85.

4. ELISA Z, et al. Multiple Congenital Colonic Stenosis: A Rare Gastrointestinal Malformation. Case Rep Pediatr, 2016; 2016:6329793.

5. GALVÁN-MONTAÑO A, et al. Congenital stenosis of the colon with foreign bodies. Case report. Cir Cir, 2010; 78(3): 259-61.

6. HAJIVASSILIOU CA. Intestinal obstruction in neonatal/pediatric surgery. Semin Pediatr Surg, 2003; $12(4): 241-253$.

7. HAMID R, et al. Management Congenital Colonic Stenosis. J Neonatal Biol, 2015; 4:166.

8. MIRZA B, et al. Colonic atresia and stenosis: our experience. J Neonat Surg, 2012; (1):4.

9. UPADHYAYA VD, et al. Colonic stenosis in neonates is not always congenital or complication of necrotizing enterocolitis. J Neonatal Surg [Internet], 2020.

10. XIE X, et al. Infant progressive colonic stenosis caused by antibiotic-related Clostridium difficile colitis-a case report and literature review. BMC pediatrics, 2018; 18(1):320. 